\title{
Knuckle Pads
}

National Cancer Institute

\section{Source}

National Cancer Institute. Knuckle Pads. NCI Thesaurus. Code C3928.

Benign skin fibromas, typically occurring over the small joints of the hands and feet, that are associated with repetitive friction or pressure. 